\title{
EFFECT OF CHARGE GRAIN COMPOSITION ON RHEOLOGICAL CHARACTERISTICS AND STRUCTURE OF PRESSURE FLOW OF COMPOUNDS FOR LOW-HYDROGEN ELECTRODES
}

\author{
A.E. MARCHENKO \\ E.O. Paton Electric Welding Institute, NASU \\ 11 Bozhenko Str., 03680, Kiev, Ukraine. E-mail: office@paton.kiev.ua
}

\begin{abstract}
It is determined in course of investigation of compound of low-hydrogen electrodes UONI-13/55, carried out with the help of capillary viscosimeter, that their rheological indices and structure under flow pressure condition significantly depend on grain composition of coating materials. Charge should contain $50 \%$ of fine fraction from point of view of minimizing of energy consumption, necessary for extrusion application of compounds on rods. Deviation of its portion in one or another side from indicated optimum significantly rises energy consumption on electrode extrusion. The compounds with fine- or coarse-grain filler are not similar to each other on structure. It confirms nature of change of level of compound dissipative heating, value of natural convergence angle in entrance zone (to capillary) as well as shape of deformation (extrusion) curves at increase of flow velocity. Profile of flow of compounds with coarse-grain filler expands at rise of pressure jet rate. It remains virtually of the same narrow shape as at creep flow velocities for flow of compounds with fine-grain filler. The results of analysis of form of extrusion curves $P=f(t)$ indicate that pressure flow of compound with coarse-grain filler is realized on viscosity mechanism. The compounds with excessive content of fine-grain filler are more structured, since liquid glass binder in these cases in addition to filling of intergrain voids should cover significantly more developed grain surface. Rise is observed in their molecular interaction and strength of structure formed by them, which is fractured in deformation and accompanied by specific phenomena of unsteady flow. 8 Ref., 2 Tables, 8 Figures.
\end{abstract}

Key words: low-hydrogen welding electrodes, coating thickness difference, rheology of compounds, viscosity and elasticity indices of compounds

Grain composition of coating materials significantly effects consistency and technological properties of electrode compounds. It is confirmed by results of investigation of viscosity of UONI-13/55 compound, published in [1, 2]. It was evaluated as pressure loss in pumping of compound from feed cylinder of viscosimeter in round nozzle of $5 \mathrm{~mm}$ diameter and $50 \mathrm{~mm}$ length at constant consumption $Q=5 \mathrm{~cm}^{3} \cdot \mathrm{s}^{-1}$ (average gradient of shear rate $100 \mathrm{~s}^{-1}$ ). Powders for dry charge were composed of preliminary screened fractions of materials in order to receive continuous packing of particles with two earlier selected indices of dispersion and polydispersity for each of them. Afterwards, prepared in such a way powders were taken in proportions, specified in mathematic plan of experiment so that grain composition of charge was changed in the limits, which can be found in practice of electrode production, i.e. volume fraction of particles finer than $0.063 \mathrm{~mm}$ in the charge was varied in the

(C) A.E. MARCHENKO, 2014 limits from 5 to 95 vol.\%, at that its specific surface varied from 3000 to $12,000 \mathrm{~cm}^{-1}$.

As a results, grain compositions of one part of charge specimens was continuous, and another one has random particle package. It was a reason for detection of number of grain compositions, providing minimum for given series of experiment pressure loss, value of which is changed at transfer from one series of compositions to another. At the same time, even small deviation of grain composition of the mixture in one or another side from each of the optimum grain compositions is accompanied by rapid, as a rule, almost symmetric increase of compound viscosity. And only series, relating to the field of coarse- and fine-grain compositions, have deviation of grain composition from the optimum accompanied by asymmetric rise of compound viscosity. In other words, identical rise of portion of fine-grain fraction in the charge in comparison with the optimum is accompanied by significantly smaller rise of compound viscosity in the first case than in the second [2]. The reason of this effect is not determined.

Study [3] investigated liquid glass compositions of marble powder with similar on width range of grain compositions (pass through mesh 


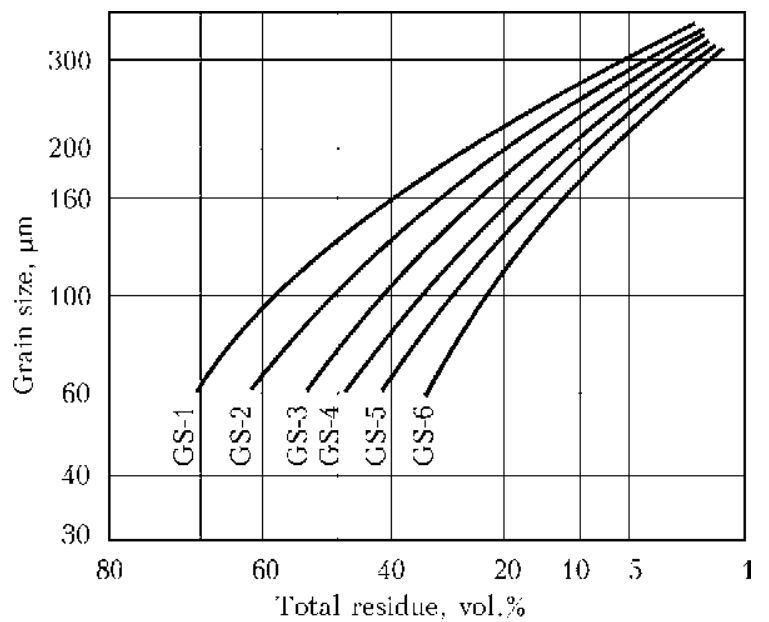

Figure 1. Grain compositions of dry charge mixtures used for production of electrode compounds GS-1-GS-6

0063 was changed in the range from 0 to 94 wt.\%, and specific surface varied from 1500 to $\left.11,500 \mathrm{~cm}^{-1}\right)$. In this series of experiments grain compositions of powders were characterized by three levels of course and width of distribution of particles by sizes, and packings of all particles, were continuous. Compositions on consistency correspond to real compounds at content of $30 \%$ of liquid glass with M 3.2 modulus and viscosity $670 \mathrm{mPa} \cdot \mathrm{s}$. Round nozzles of diameter/length $4 / 20 \mathrm{~mm}$ at $Q=1 \mathrm{~cm}^{3} \cdot \mathrm{s}^{-1}$ or $8 / 60 \mathrm{~mm}$ at $Q=$ $=5 \mathrm{~cm}^{3} \cdot \mathrm{s}^{-1}$ (average gradient of shear rate 40 and $25 \mathrm{~s}^{-1}$, respectively) were used as measuring instrument.

This series of experiments showed one relatively wide minimum of viscosity, which falls on $30-60 \%$ of fine fraction in the charge. Position of minimum is constant in studied range of flow velocities. However, flow velocity significantly effects viscosity of extreme (the most coarse- and fine-grain) specimens of marble. At that, the same decrease of shear rate from 40 to $25 \mathrm{~s}^{-1}$ is accompanied by rise of viscosity of suspension with coarse-grain filler and its reduction in suspension with fine-grain filler. It can be assumed that such suspensions, characterized approximately by similar fractional void space (free in-

Table 1. Characteristics of charge and compound

\begin{tabular}{|c|c|c|c|c||}
\hline $\begin{array}{c}\text { Designation } \\
\text { of compound }\end{array}$ & $\begin{array}{c}\text { Portion of } \\
\text { fine } \\
\text { fraction, } \%\end{array}$ & $\begin{array}{c}\text { Specific } \\
\text { surface of } \\
\text { charge, } \mathrm{cm}^{-1}\end{array}$ & $\begin{array}{c}\text { Maximum } \\
\text { allowable } \\
\text { concentration } \\
F_{m}\end{array}$ & $\begin{array}{c}\text { Plastic } \\
\text { strength } P_{m} \\
\text { MPa }\end{array}$ \\
\hline GS-1 & 26 & 2250 & 0.720 & 0.13 \\
\hline GS-2 & 36 & 3900 & 0.800 & 0.10 \\
\hline GS-3 & 43 & 4900 & 0.815 & 0.12 \\
\hline GS-4 & 47 & 5850 & 0.815 & 0.13 \\
\hline GS-5 & 56 & 8100 & 0.750 & 0.22 \\
\hline GS-6 & 65 & 9250 & 0.705 & 0.28 \\
\hline
\end{tabular}

tergranular space) of filler, have at the same time different structure.

In general, the results obtained in the works indicated above can be well explained from point of view of hydrodynamic theory of viscosity based on matching of real density of packing of particles of filler $F$ with their maximum allowable concentration $F_{m}$ reaching, which the suspension loses flow capability. Significant part of binder becomes kinetically free liquid as a result of reduction of fractional void space, typical for monodisperse filler due to filling of the voids by finer particles. It simplifies shear movement of grains relatively each other, i.e. suspension viscosity is reduced.

Aim of the present work is an investigation of effect of charge grain composition on rheological characteristics and structure of pressure flow of compounds for low-hydrogen electrodes at flow velocities, corresponding to real conditions of electrode extrusion (application of compound) using extrusion presses.

Procedure of investigation. Rheological characteristics of pilot compound having the following component characteristics of dry charge, wt.\%: 51 marble, 18 fluor-spar concentrate, 5 quartz sand, 3 synthetic mica, 2 ferromanganese, $13(15 \% \mathrm{Si})$ granulated ferrosilicon and 8 ferrotitanium, were investigated. Grain composition of charge was regulated by means of changing of proportion of weight fractions of preliminary screened powder fractions of marble, fluorspar and quartz sand. Powders of ferro-alloys and synthetic mica ANS-1 were used with constant grain composition. General proportion of fine fraction in the charge was varied in the limits from 25 to $65 \mathrm{wt} . \%$, at that proportion of fractions was changed in such a way as shown in Figure 1. Grain composition of mixture with the most compact packing of grains, corresponding to Furnas rule, lies in the range between curves GS-4 and GS-5. Real indices of specific charge surface and density of their random packing as well as plastic strength of the compounds are given in Table 1.

Compounds were prepared in intensive counter-flow mixer. $\mathrm{Na}-\mathrm{K}$ liquid glass having M 2.9, $1495 \mathrm{~kg} / \mathrm{m}^{3}$ density and $1000 \mathrm{mPa} \cdot \mathrm{s}$ viscosity was used. Weight fraction of liquid glass in the compound makes $25 \%$.

Investigations were carried out using capillary viscosimeter of OB-1435 model, representing itself plunger extruder $[2,4]$ with electro-mechanical drive and diameter of operating cylinder $30 \mathrm{~mm}$. Using stepwise change of march rate of piston, per second consumption of compounds was regulated in the range $Q=1-25 \mathrm{~cm}^{3} \cdot \mathrm{s}^{-1}$. 
Using of round nozzles ( «capillary») with flat outlet and diameter $d_{\mathrm{c}}=1-6 \mathrm{~mm}$ at such consumptions allowed regulating average gradient of shear rate on smooth wall of channel in the ranges from 10 to $65,000 \mathrm{~s}^{-1}$.

Shear stress on channel wall $\tau$ was calculated on formula $P d_{\mathrm{c}} / 4 L$, where $L=10 d_{\mathrm{c}}$ is the length of channel; $P=\left(P_{\mathrm{c}}-P_{0}\right)$ is the drop of pressure at this length; $P_{0}$ is the pressure loss at inlet to the nozzle (determined by means of passing of the compound through round hole of $d_{\mathrm{c}}$ diameter in the center of steel disk of $1 \mathrm{~mm}$ thickness); $P_{\mathrm{c}}$ is the general loss of pressure before inlet to the nozzle and over its length.

Similar level of thixotropic fracture (reconstruction) of coagulation structure of compounds for all nozzle diameter was maintained by $L / d_{c}$ constant relationship. Duration of compound extrusion was varied depending on flow velocity in 5-15 s range, and $P_{\mathrm{c}}$ and $P_{0}$ values were registered at the moment of piston stop. The capillaries are not temperature-controlled. Thermalcouple, calked in the body of capillary of $4 \mathrm{~mm}$ diameter and $56 \mathrm{~mm}$ length, was used for sampling testing of temperature of jet surface at $Q=$ $=1 \mathrm{~cm}^{3} \cdot \mathrm{s}^{-1}$. Thermoelectromotive force was registered using potentiometer KSP-4. The experiments were carried out by Dr. Gnatenko M.F. and Eng. Voroshilo V.S.

Efficient shear viscosity of the compound in pressure flow state was calculated on formula
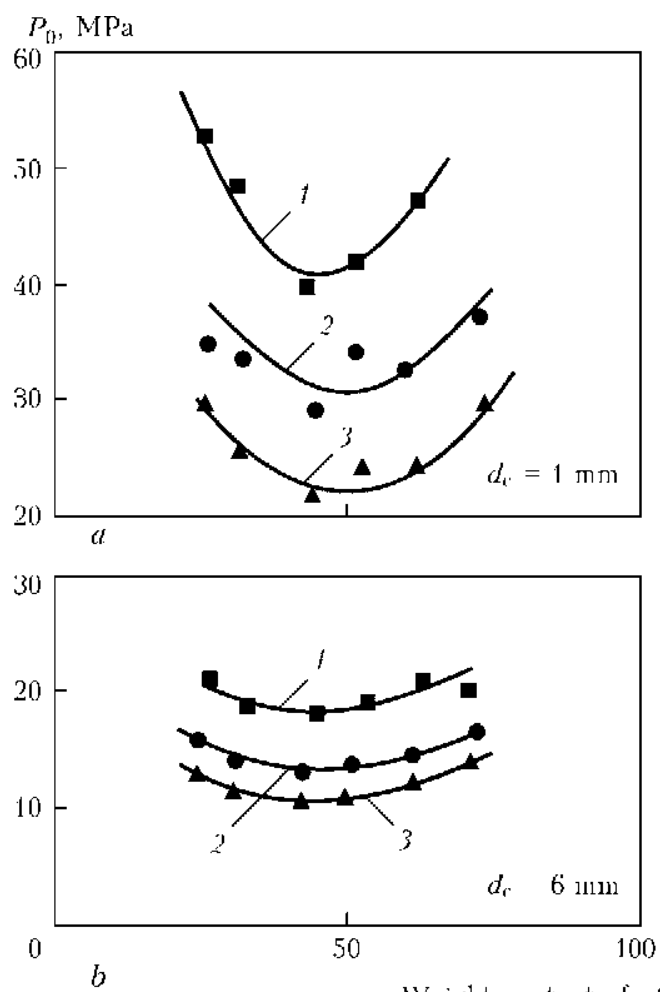

Weight content of -0063 fraction in charge, \% $\eta=\tau / \dot{\gamma}$ and longitudinal viscosity $\lambda$ was received using the following formula from works $[5,6]$ :

$$
\lambda=\frac{9\left(n_{0}+1\right)^{2}}{32 \eta}\left(\frac{P_{0}}{\dot{\gamma}}\right)^{2},
$$

where $n_{0}=d\left(\lg P_{0}\right) / d(\lg \dot{\gamma})$ is the index of compound flow in convergent zone, which was determined by angle of inclination of rheograms $P_{0}=f(\dot{\gamma})$ to axis of shear rate gradients, represented in logarithmical coordinates. Similar on meaning index of shear flow of compound through cylinder channel $n_{\mathrm{c}}=d(\lg \tau) / d(\lg \dot{\gamma})$ was also used. Both indices characterize relationship of energy of activation of material viscous flow at $\dot{\gamma}=$ const and $\tau=$ const, respectively $n_{\mathrm{c}}<$ $<n_{0}$ as a rule.

Structure of compound flow was estimated on value of angle of natural convergence $\alpha_{0}$, which corresponds to equality of shear and extension components of force, overcoming resistance of indicated zone [6].

Angle $\alpha_{0}$ reduces with rise of flow velocity for those materials, where longitudinal viscosity decreases intensively than shear one. Convergent zone of such materials acquires watering-can shape [1, 2, 4, 5].

The following formula was used for determination of average compound extension stress:

$$
\left(\sigma_{E}\right)_{\mathrm{av}}=\frac{3}{8}\left(n_{0}+1\right) P_{0},
$$
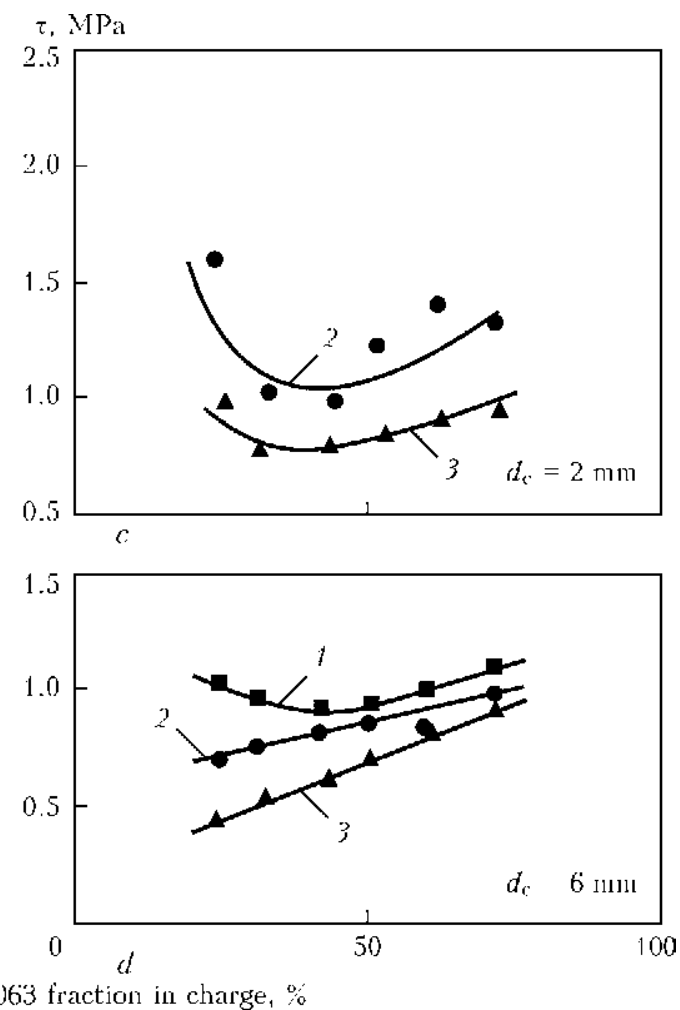

Figure 2. Effect of fine fraction in charge on pressure loss at inlet $(a, b)$ and shear stress on capillary wall $(c, d): 1-$ $Q=25.5 \mathrm{~cm}^{3} \cdot \mathrm{s}^{-1} ; 2-5.3 ; 3-1.0$ 
and gradient of extension rate was calculated on formula

$$
\dot{\varepsilon}=\frac{\left(\sigma_{E}\right)_{\mathrm{av}}}{\lambda} .
$$

Angle of natural convergence at the inlet of compound in shaping cylinder nozzle was estimated on formula

$$
\operatorname{tg} \alpha_{0}=\left(\frac{2 \eta}{\lambda}\right)^{1 / 2}
$$

Results of investigation and their discussion. Figures 2 and 3 show determined in course
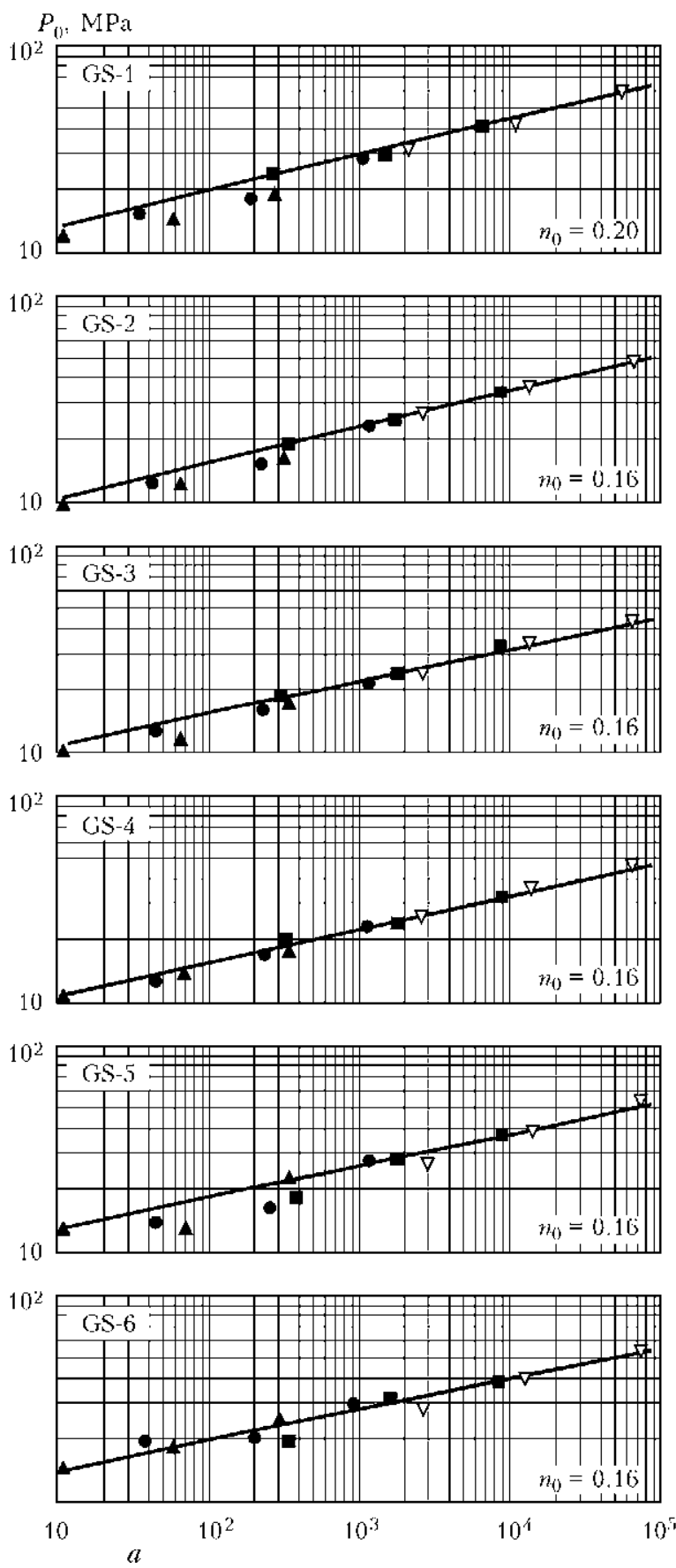

Figure 3. Dependence of gradient of shear rate on inlet resistance $(a)$ and shear stress on capillary wall $(b)$ on portion of fine fraction in compound: $\boldsymbol{\Delta}-d_{\mathrm{c}}=6 \mathrm{~mm} ;-4 ; \mathbf{\square}-2 ; \nabla-1$

of experiments dependencies of input resistances $P_{0}$ and shear stress on wall of cylinder nozzle $\tau$, received using the nozzles with extreme sections of channels, on portion of fine fraction in the charge, as well as their dependence on shear rate gradient on channel wall. It can be seen that charge grain composition, changing even in the range of such narrow limits, significantly effects rheological characteristics of the compounds. Particularly, if we are taking on compound flow resistance in convergent zone. As it is was predicted, $P_{0}$ value is varied on extreme law in dependence of portion of fine fraction in the charge
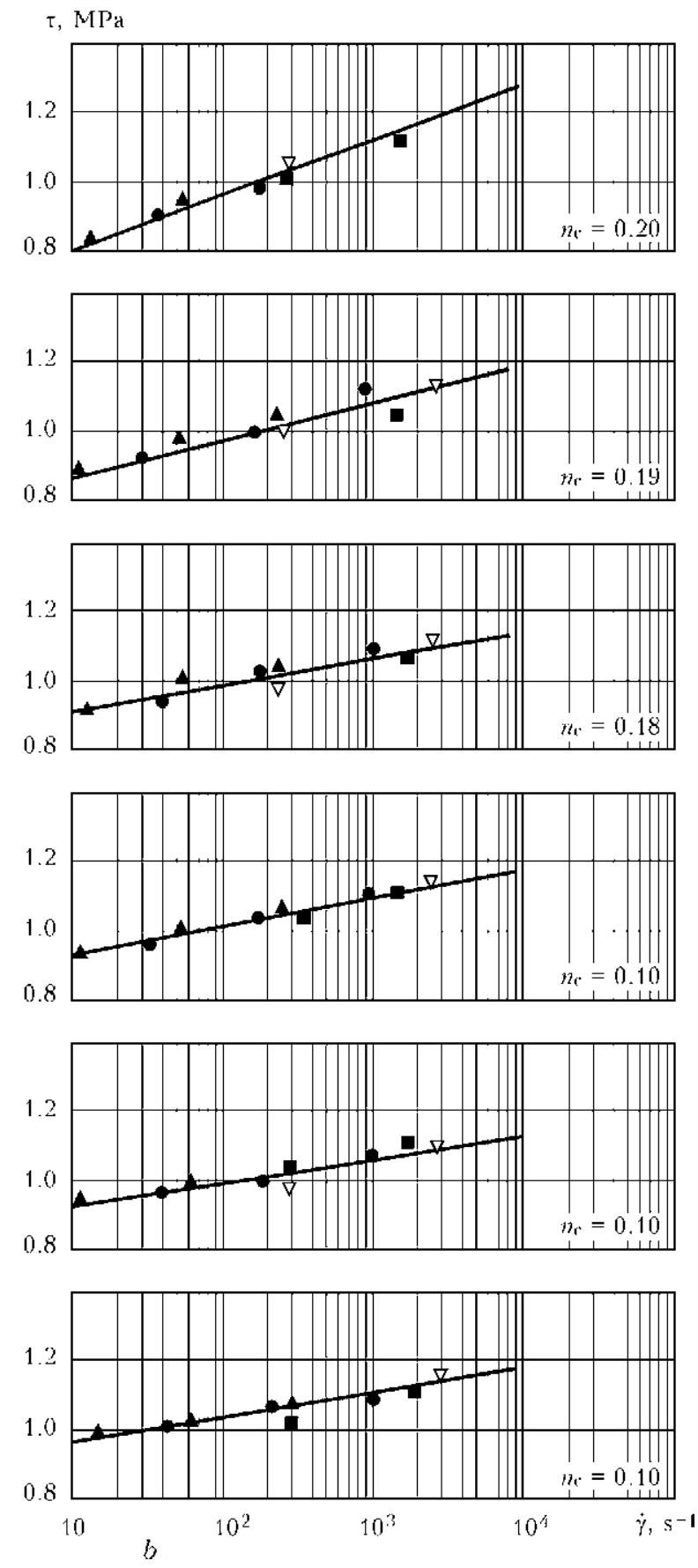
and significantly rises with its deviation in one or another side from $50 \%$ being the optimum value. The smaller the section of outlet hole and the lager the volume consumption of the electrode compound, the greater is the reaction of compound on change of charge grain composition, when overcoming input resistance in shaping cylinder channel. Nevertheless, only the most fine-grained among them (65\% of fine fraction in charge) do not pass through diaphragm of $1 \mathrm{~mm}$ diameter at $Q=25.5 \mathrm{~cm}^{3} \cdot \mathrm{s}^{-1}$. The rest of compounds passed through this and other diaphragms at all volume flow velocity.

Behavior of compounds in cylinder nozzles is more complex. First of all, any of compounds overcame resistance of nozzles with channels of 1 and $2 \mathrm{~mm}$ diameters at $Q=25.5 \mathrm{~cm}^{3} \cdot \mathrm{s}^{-1}$. Part of compounds passed through the nozzles of $2 \mathrm{~mm}$ diameter, if consumption did not exceed $5.1 \mathrm{~cm}^{3} \cdot \mathrm{s}^{-1}$. Nozzle with channel diameter $6 \mathrm{~mm}$ passed through all the compounds at all consumptions and charge grain compositions. Secondly, extreme variation of shear stress on nozzle wall depending on portion of fine fraction in the charge is less expressed than in inlet resistances. Extreme $\tau$ dependence on portion of fine fraction in the charge degenerates into monitonically rising at flow modes with 1 and $1.5 \mathrm{~cm}^{3} \cdot \mathrm{s}^{-1}$ consumption, when using nozzles with channel diameter 4 and $6 \mathrm{~mm}$. These peculiarities cannot be explained considering provisions of only hydrodynamic viscosity theory.

It is important to be noted that all compounds behave themselves as materials with pronounced non-Newtonian properties. It is indicated by values of flow indices, which are significantly smaller than one, namely in convergent zone $n_{0}=$ $=0.16$ independent on charge grain composition; in capillary $n_{\mathrm{c}}$ monotonically reduces from 0.2 to 0.1 with increase of portion of fine fraction in the charge. Therefore, non-Newtonian nature of compounds is more pronounced.

Change of charge grain composition influences the structure of flow in the entrance zone as well as cylinder nozzle. The results given below show that increase of portion of fine fraction in the charge provides for change of values of longitudinal and shear viscosity and together with them variation of convergence angle in the entrance zone, which, as follows from (4), is determined by their relationship. At that, tendency to slug nature of compound flow (index of flow is reduced) in the nozzle should intensify due to what shear is more and more concentrated in near-wall layer.

Figure 4 gives the results of calculation of shear and longitudinal viscosity of studied com-

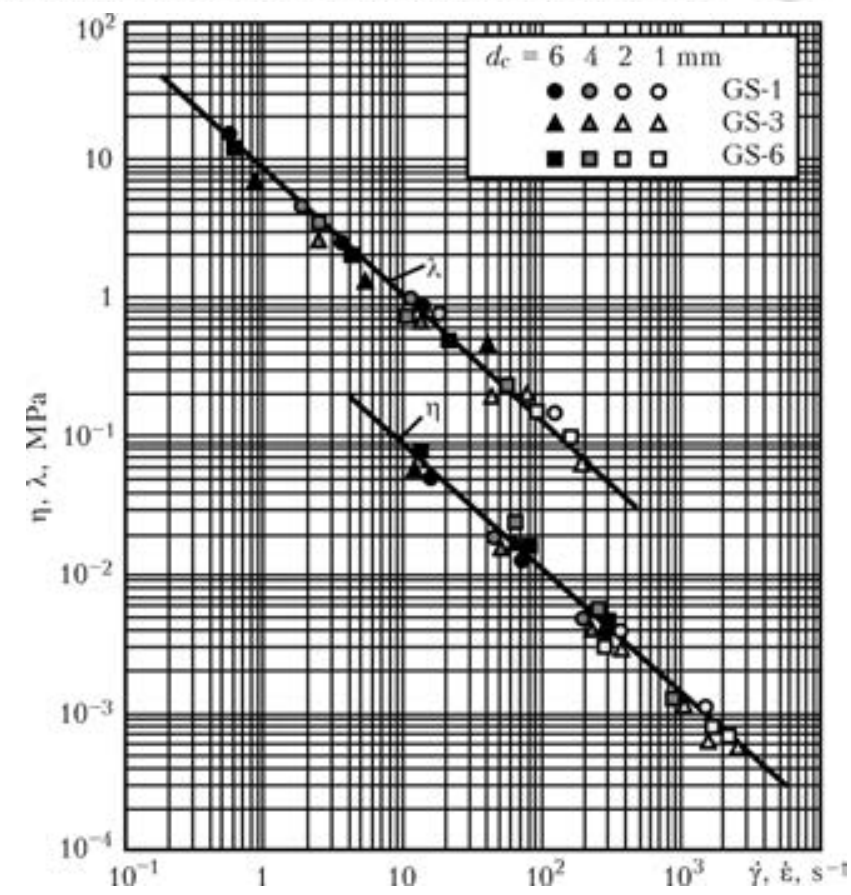

Figure 4. Dependence of shear $\eta$ and longitudinal $\lambda$ viscosity of electrode compounds with different charge grain composition on average gradient of shear rate $\dot{\gamma}$ and extension $\dot{\varepsilon}$

pounds depending on shear rate gradient and extension, respectively. It can be observed that $\eta$ and $\lambda$ reduce with rise of deformation rate. This confirms good structure of electrode compounds and thixotropic fracture of their coagulation structure in rise of $\dot{\gamma}$ and $\dot{\varepsilon}$. It also can be seen that experimental points in logarithmic metamorphosis can be well matched with straight lines, generalizing interesting for us dependencies. The $\eta=f(\dot{\gamma})$ and $\lambda=f(\dot{\varepsilon})$ straight lines itself are almost mutually parallel and only being shifted relatively to each other on scales of gradients of shear and extension, respectively. It is assumed that charge grain composition has small effect on relationship of values of shear and longitudinal viscosities (and so, on profile of compound flow in the entrance zone).

In fact, close to parallel behavior of rheograms $\eta=f(\dot{\gamma})$ and $\lambda=f(\dot{\varepsilon})$ in logarithmic coordinates does not indicate at all a consistency of $\lambda / \eta$ viscosity relationship. It was determined in the following way. Firstly, relationship of shear and longitudinal viscosities of compounds was determined for each mixture grain composition. Examples of such dependencies for three grain compositions with extreme and average portion of fine fraction in the charge are shown in Figure 5. Afterwards, inclination of straight lines $\lg \lambda / \lg \eta$ to abscissa axis was evaluated. It firstly decreases with increase of portion of -0063 fraction in the charge, and it rises after reaching the minimum value (Figure 6). In this connec- 

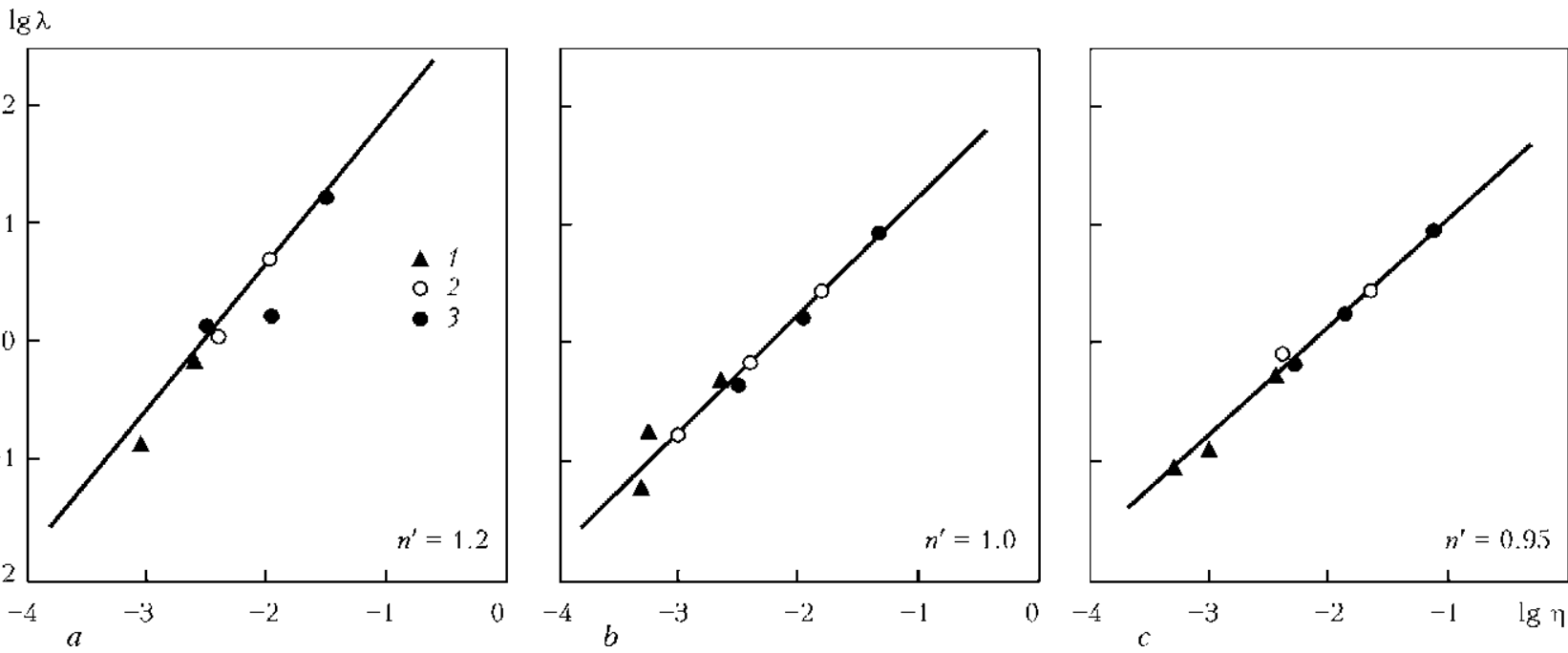

Figure 5. Relationship of longitudinal ( $\lambda$, MPa.s) and shear ( $\eta$, MPa.s) viscosity of electrode compounds GS-1 (a), GS-4 (b), GS-5 (c) with different charge grain composition: $1-d_{\mathrm{c}}=6 \mathrm{~mm} ; 2-4 ; 3-2$

tion, the value of convergence angle $\alpha_{0}$ shows ambiguous reaction on change of charge grain composition and compound flow modes. It follows form Figure 7 that low and average gradients of shear rate also promote change of convergence angle depending on portion of fine fraction in the charge based on extreme law (it nature is in $P_{0}$ inverse relation on portion of fine fractions in the charge, and maximum falls at $50 \%$ of fine fraction).

In this case, fine- and coarse-grain filler promotes narrow profiles of compound flow, that can result in formation of leading outflow of its internal layers in comparison with external layers. Usually, the narrow flow profiles provide for pulsing and twisting of the jet, i.e. position of such flow in principle can not be stably oriented in space. Using of charges with intermediate grain having, as a rule, the widest grain size distribution, promotes formation of more distributed and, it can be assumed, more stably oriented in space flow at low velocities.

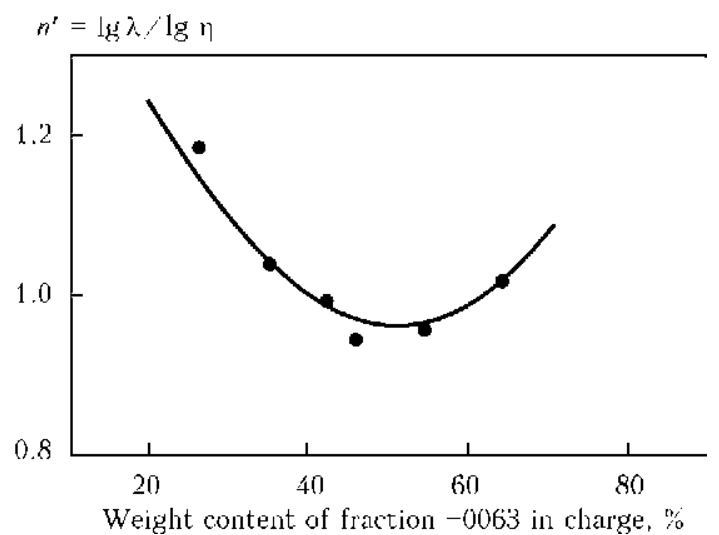

Figure 6. Effect of charge grain composition on relationship of longitudinal and shear viscosities of electrode compounds of GS series
Rise of shear rate gradient provides for gradual evening of curve maximums, and indicated dependencies become monotonic at $(1-2) \cdot 10^{3} \mathrm{~s}^{-1}$ gradients, which are predicted for compound flow velocities under real conditions of electrode extrusion. At that, the compounds with coarsegrain filler, in which portion of fine fraction makes 20-25 wt.\%, form wider flow profiles. The flow with such profile should have more stable orientation in space. Advancing flow of material in core and, respectively, appearance of periphery dead zones are less probable in it.

Compounds with average- and, in particular, fine-grain filler (in which portion of -0063 fraction makes 40 and 60-65 wt.\%, respectively) almost preserve initial profile of flow velocities in these modes. The reasons of such changes can be related with non-isothermal flow conditions as well as different structure of compared compounds.

Table 2 shows that their dissipative heating in creep flow mode $\left(Q=1 \mathrm{~cm}^{3} \cdot \mathrm{s}^{-1}\right)$ is insignificant (to $34-37^{\circ} \mathrm{C}$ ) and the same for all. It rarely can effect viscosity characteristics of the compounds and does not allow determining their structural peculiarities. Increase of shear rate gradient promotes for heating of the compounds to higher temperatures, compound GS-4 is the most intensive. Since portion of kinetically free liquid glass

Table 2. Results of estimation of temperature condition in compound flow zone at $4 \mathrm{~mm}$ nozzle diameter

\begin{tabular}{|c|c|c|c|c||}
\hline \multirow{2}{*}{$\begin{array}{c}\text { Compound } \\
\text { consumption, } \\
\mathrm{cm}^{3} \cdot \mathrm{s}^{-1}\end{array}$} & \multirow{2}{*}{$\begin{array}{c}\text { Gradient of } \\
\text { shear rate, } \\
\mathrm{s}^{-1}\end{array}$} & \multicolumn{3}{|c|}{ Temperature of jet, ${ }^{\circ} \mathrm{C}$} \\
\cline { 3 - 5 } & 11.8 & 34 & 37 & GS-1 \\
\hline 1.0 & 203 & 58 & 54 & 53 \\
\hline 5.1 & 1015 & 76 & 91 & 77 \\
\hline 25.5 & \multicolumn{3}{|l}{} \\
\hline
\end{tabular}




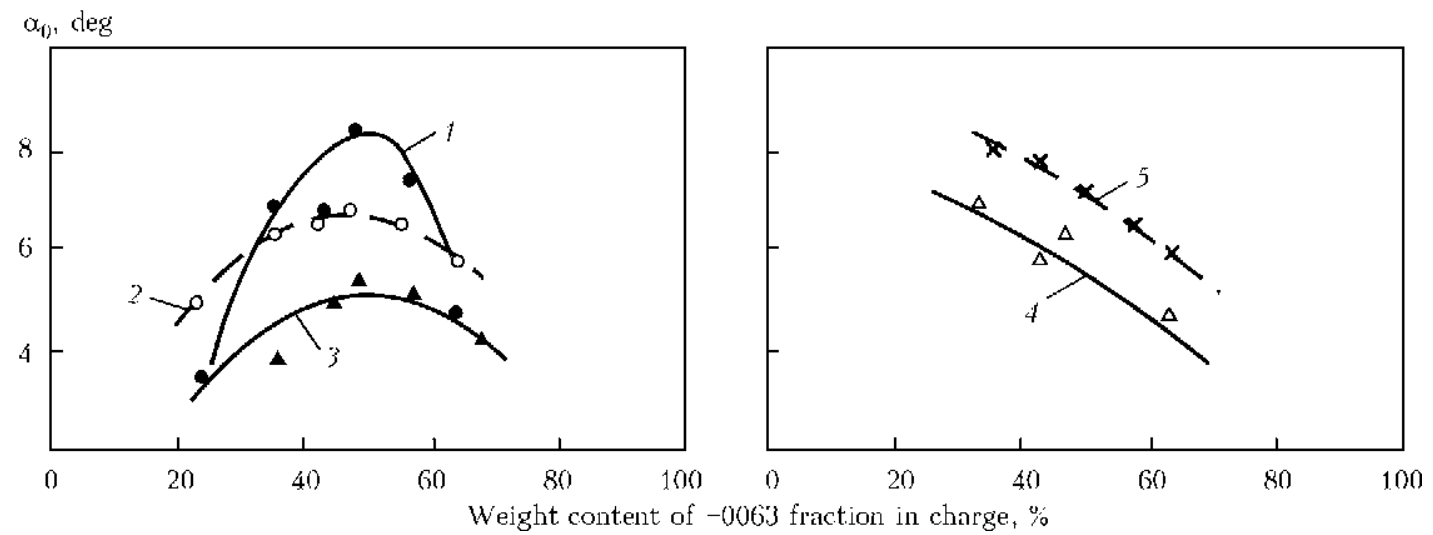

Figure 7. Effect of charge grain composition and flow velocity of compound on value of natural convergence angle of its pressure flow in pre-capillary zone: $1-\dot{\gamma}=118 \mathrm{~s}^{-1} ; 2-203 ; 3-318 ; 4-1015 ; 5-2550$

in intergrain space of this compound is the largest, it less structural than GS-1 and GS-6 compounds. Energy consumptions for structure fracture do not mask its dissipative heating, and this is a reason of so rapid rise of temperature. GS-1 with coarse-grain filler is the next compound on level of structuring. It is heated to $76{ }^{\circ} \mathrm{C}$, but at little bit higher intermediate rate in comparison with compound GS-4. Compound GS-6 reaches the same level of temperature with the lowest intermediate rate. Respectively, it should have the highest level of structuring.

It is shown above that change of $\alpha_{0}=f(-0063)$ function of the same compounds also demonstrates ambiguous reaction on increase of shear rate. It supports an assumption made earlier that we are dealing with the materials having different rheological structures.

This is also confirmed by comparison of form of extrusion (deformation) curves $P=f(t)$, given in Figure 8, and received during study of different compounds. Indicated curves describe the changes of pressure from moment of viscosimeter start, including reaching the maximum, consequent drop, promoted by compound flow, fracture of its structure and relaxation of accumulated stresses, up to setting of pressure corresponding to steady compound flow.

It should be considered in this case that rate of compound deformation in course of extrusion at constant consumption per second rises with decrease of capillary diameter. Shape of specified curves indicates that matched compounds should differ between themselves by relationship of viscosity and elasticity, accumulated in process of extrusion [7].

It follows from Figure $8, b$ that compound GS-4 is characterized by constant rate of pressure rise in time independent on deformation rate. It is only one of three compounds capable to overcome resistance of capillary of $1 \mathrm{~mm}$ diameter at
$Q=25.5 \mathrm{~cm}^{3} \cdot \mathrm{s}^{-1}$. According to provisions of viscoelasticity theory, it indicates low capability of material for accumulation of elastic stresses in course of pre-stationary stage of pressure flow.

The higher the extrusion rate, the more is the rate of pressure rise after deformation beginning in compounds GS-1 and GS-6 with excessive content of coarse- and fine-grain filler. Therefore, they more intensively accumulate elastic stresses and reduce portion of net energy, which is consumed for compound extrusion, at rise of extrusion rate.

Compound flow is accompanied by stress relaxation. The higher the set rate of deformation, the lager is the level of pressure rise, outrunning

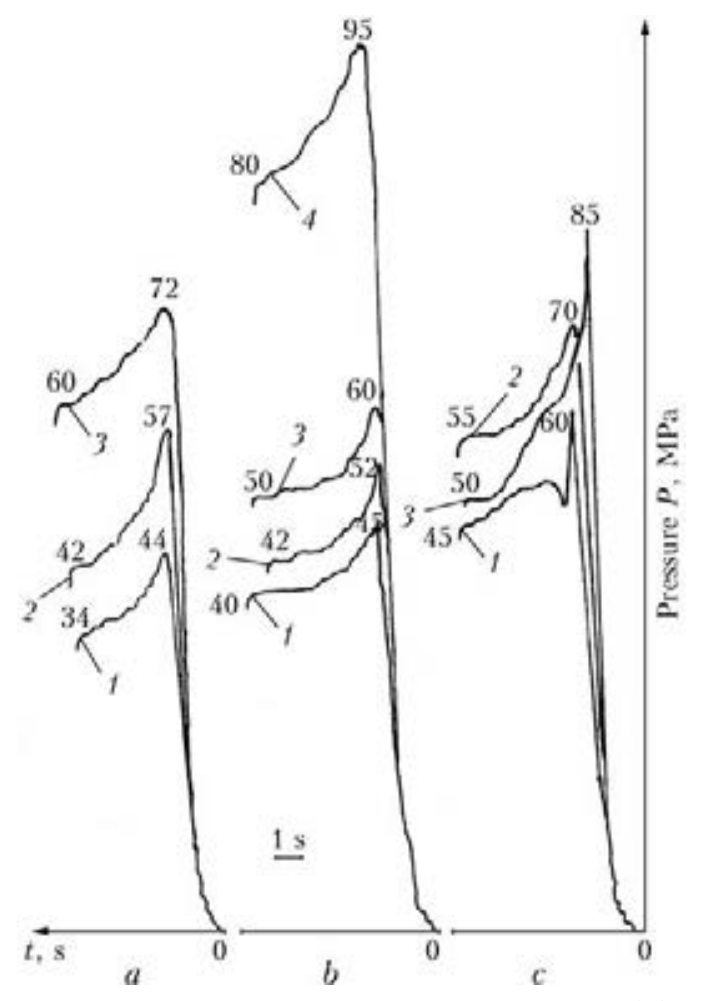

Figure 8. Curves of extrusion of compounds GS-1 (a), GS-4 (b) and GS-6 (c) with different quantity of fine fraction in charge passing through capillaries of $6(1), 4(2), 2$ (3) and 1 (4) $\mathrm{mm}$ diameter at $Q=1 \mathrm{~cm}^{3} \cdot \mathrm{s}^{-1}$ 
the rate of stress relaxation, after deformation beginning. This in particular intensifies accumulation of the elastic deformations. This process is more pronounced in compound GS-6 with finegrain filler. It is shown by almost linear rate of pressure rise, on the one hand, and sharp peak of pressure in maximum, on the other hand. Compound GS-1 is less structured, therefore, rising branch of extrusion curve coming to peak significantly deviates from the straight line, that is caused by overlaying of viscosity flow to elastic deformation.

Relaxation of the accumulated stresses in compared compounds takes place in different way after passing the maximum. The relaxation of momentary elastic stresses in compound GS-6 extruded at low rates (Figure 8, c, curve 1) provokes short, but rapid pressure drop. After drop it is partially recovered and then continues smooth reduction to receiving of steady flow. Pressure in maximum is smoothed and structural branch is reduced without intermediary drop at average rate of extrusion (Figure 8, $c$, curve 2). Level of pressure under condition of steady flow is more than at previous curve. Structural branch of the curve at the highest flow velocity ( $F i-$ gure $8, c$, curve 3 ) after sharp maximum shows so intensive reduction that it falls below than in the similar curve, registered at intermediate extrusion rate. Namely, this anomaly of deformation curves is usually related with high structuring of coagulation dispersions. Compounds GS-1 and GS-4 have no such anomaly.

The lager level of structuring of GS-6 compound in comparison with GS-1 compound is explained in the following way. Increase of portion of fine fraction above the optimum values in the charge provides for rise of not only portion of intergrain voids (and, respectively, reduction of quantity of kinetically free binder from liquid glass), but also specific surface of the particles, which should be covered by binder. Thus, the system as though is transferred in the condition with lager space filling, i.e. higher concentration of solid particles contacting with each other. Considering indicated factors, thickness of intergrain film is reduced and molecular interaction of the filler particles, being the most intensive in the points of their interaction, is significantly risen. It is verified by increase of strength of coagulation structure $P_{m}$ (see Table 1).

Results of our experiments match well with calculations carried out in work [8]. The latter show that position of viscosity minimum of suspension with multimodal filler deviates to larger extent in the side of lower concentration of coarse fractions in the filler in comparison with that, which is provided by the densest particle packing for given grain composition.

The similar effect can be achieved, if viscosity liquid glass is replaced by low-viscosity one, reducing at that its portion in compound within the due limits. In this case, elastic relaxation of GS-6 compound is not compensated by damping capability of the low-viscosity liquid glass and can promote pulsing of its flow in creep flow mode or different type irregular effects under flow modes, exceeding creep deformation on rate. The first and the second can, in particular, be the reason of coating thickness difference.

There is no pulsing of flows of GS-1 and GS-4 compounds, filler of which contains less fine fractions.

\section{Conclusions}

1. Rheological characteristics of electrode compounds were investigated depending on charge grain composition. Portion of fine fraction in the charge was varied in the limits close to that given in specification (40-60\% of particles finer than $0.063 \mathrm{~mm}$ ). It was found that charge should contain $50 \%$ of fine fraction for minimizing of consumption of energy necessary for extrusion application of compounds over the rods. Deviation of its content in one or another side from the indicated optimum, even in such narrow limits, significantly rises consumption of energy for electrode extrusion, in particular, at rates which are used under real conditions of their manufacture.

2. Such dependence from point of view of hydrodynamic theory is explained by increase of compound viscosity, caused by rise of void free space between filler grains, which should be filled by liquid glass with certain excess, before the compound will gain a capacity to pressure flow. Filling of the voids between the filler coarse grain by fine particles promotes displace of liquid glass from them, transforming it in kinetically free liquid. This results in reduction of compound viscosity.

3. The compound containing excessive quantity of coarse or fine fractions in comparison with the specified optimum are not similar to each other. It is indicated by nature of change of compound temperature, value of convergence angle, which is formed in the entrance zone as well as shape of extrusion curves at rise of deformation rate. Flow profile of compounds with coarse grain filler expands with rise of pressure jet rate, and this promotes its stabilizing in space and time. Flow of the compound with fine-grain filler is almost the same narrow as at creep flow veloci- 
ties, that is unfavorable moment from technological point of view.

4. The results of analysis of shape of extrusion curves $P=f(t)$ indicate that pressure flow of the compound with equal portions of coarse- and finegrain filler takes place on viscosity mechanism with the lowest energy consumption. Flow of the compound with excessive content of coarse-grain filler is also performed on viscosity mechanism, but with larger viscosity, since glass is often used for void filling. Their viscosity is higher than in compounds with equal portions of coarse- and fine-grain filler, since part of liquid glass is consumed for filling of intergrain voids of the filler, volume of which in this case is larger, as necessary quantity of fine particles for their filling is absent. The compounds with excessive content of fine-grain filler are more structured, since liquid glass binder in addition to filling of intergrain voids should cover significantly more developed surface of fine grains. The rise is observed in molecular interaction of filler grains and strength of structure formed by them, which can be easily fractured in shear deformation, accompanied by specific effect of unsteady flow.

5. Consideration of peculiarities of electrode compound pressure flows with high deformation rates should take into account the peculiarities of coagulation structures formed by them and their reaction on change of deformation rate in addition to provisions of hydrodynamic theory of viscosity of highly concentrated dispersion systems.

1. Marchenko, A.E., Pokhodnya, I.K., Skorina, N.V. et al. (1994) Development of technology for manufacture of low-carbon electrodes. Svarochn. Proizvodstvo, 5, 14-18.

2. Marchenko, A.E. (1978) On rheological methods of evaluation of technological properties of electrode compounds: SMEA Inform. Doc., Issue 1, 121-128. Kiev: Naukova Dumka.

3. Marchenko, A.E. (2013) Examination of concentrated suspensions of marble in liquid glass as a rheological model of electrode compounds. In: Proc. of 7th Int. Sci.-Techn. Conf. on Welding Consumables. Arc Welding. Materials and Quality (Krasnodar, vil. Agoj, 17-21 June 2013), 98-115.

4. Marchenko. A.E., Gnatenko, M.F. (1980) Peculiarities of flow of electrode compounds detected by capillary plastometer: SMEA Inform. Doc., 106-117. Kiev: Naukova Dumka.

5. Marchenko, A.E. (2011) About rheological properties of electrode compounds in convergent zone during electrode extrusion. In: Proc. of 6th Int. Conf. on Welding Consumables. Development. Technology. Manufacture. Quality. Competitiveness (Krasnodar, 6-9 June 2011), 223-232.

6. Cogswell, F.N. (1972) Converging flow of polymer melts in extrusion dies. Polymer Eng. and Sci., 12(2), 64-70.

7. Belkin, I.M., Vinogradov, G.V., Leonov, A.I. (1967) Rotary devices. Measurement of viscosity and physico-mechanical characteristics of materials. Moscow: Mashinostroenie.

8. Moshev, V.V. (1977) Viscosity principles of highly filled polymers. In: Rheology (polymers and petroleum): Transact. of All-Union school on rheology, 53-64. Novosibirsk: ITF.

Received 15.04.2014 